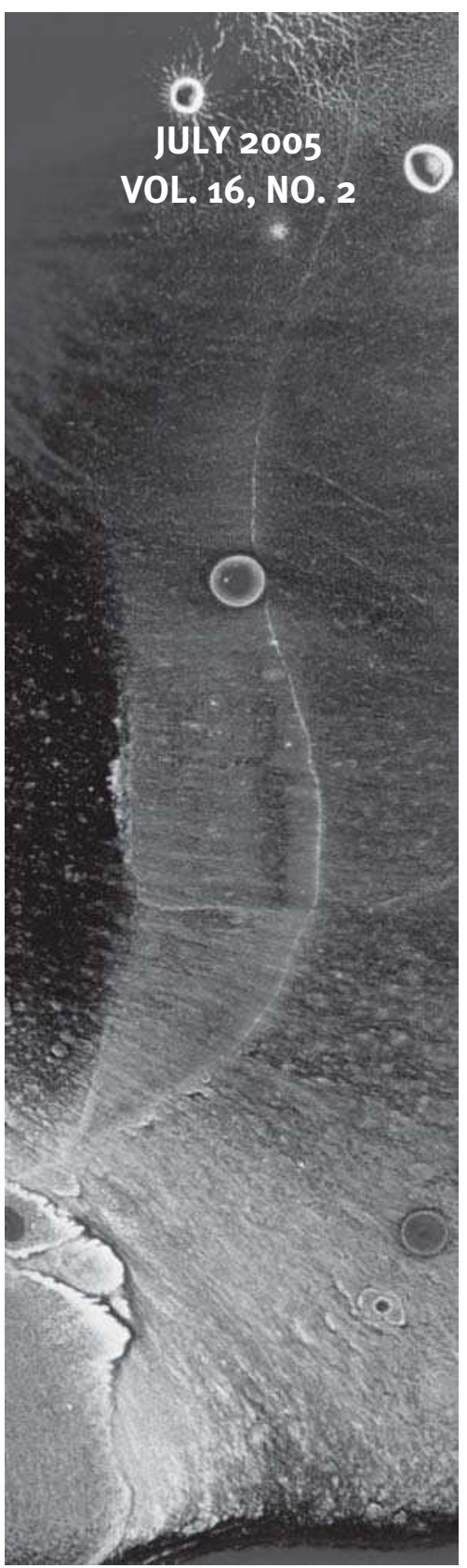

\section{SPIE International \\ Technical Group Newsletter}

\section{Calendar-See page 10.}

Technical Group Registration Form-See page 11.

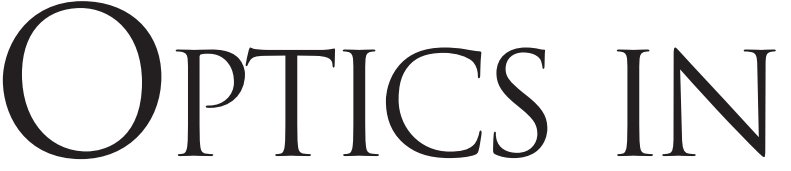

INFORMATION

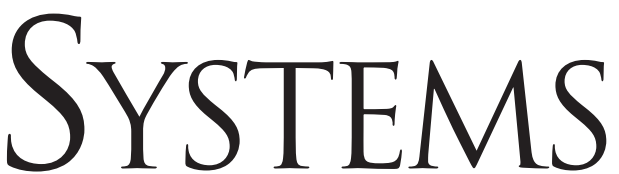

\title{
Compression of encrypted digital holo- grams using artificial neural networks
}

An important aspect of security and defense is information gathering, dissemination, processing, and analysis. Central to this is the encryption and decryption of messages for storage and transmission. Although public-key cryptosystems are the state of the art currently, there is a place for private-key systems in cases where hardware implementation permits very high throughputs. Optical implementation is a candidate for this. Optics has some very promising scalability advantages over purely electronic systems as, in principle, the size of the key can be increased without increasing the encryption or decryption time. Furthermore, optics is perfectly suited to scenarios where message distortion in the encryption/decryption process is permissible in order to increase efficiency. In such scenarios, the different processes involved in the secure transmission of image information-such

Continues on page 8 .

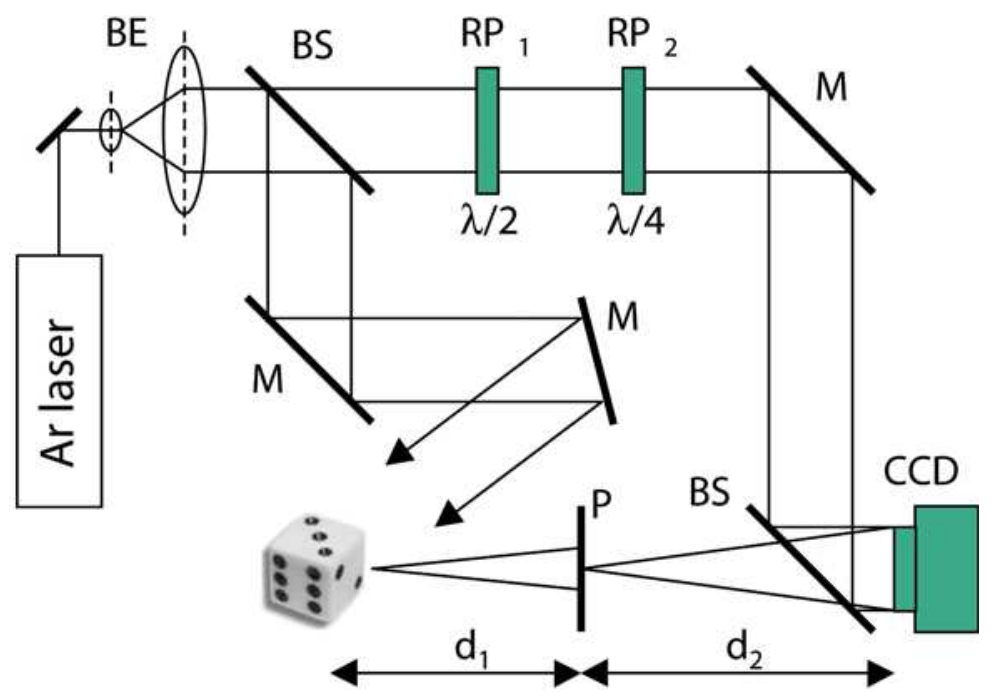

Figure 1. Experimental setup for 3D object encryption using phase-shift digital holography. BE: beam expander. BS: beamsplitter. M: mirror. RP: retardation plate. P: phase mask. 


\section{Compression of encrypted digital holograms using artificial neural networks}

Continued from cover.

as compression and encryption/decryption - can go hand in hand.

Recently, digital holography has been used in the encryption of two-dimensional images and 3dimensional (3D) objects. ${ }^{1,2}$ In this article, the complex-valued encrypted holographic pixels are quantized nonuniformly using an unsupervised artificial neural network (unsupervised ANN) to achieve lossy data compression. Two important differences between digital hologram compression and conventional image compression are that our holograms store 3D information in complex-valued pixels, and the holograms' inherent speckle content gives them a white-noise appearance. Holographic speckle is

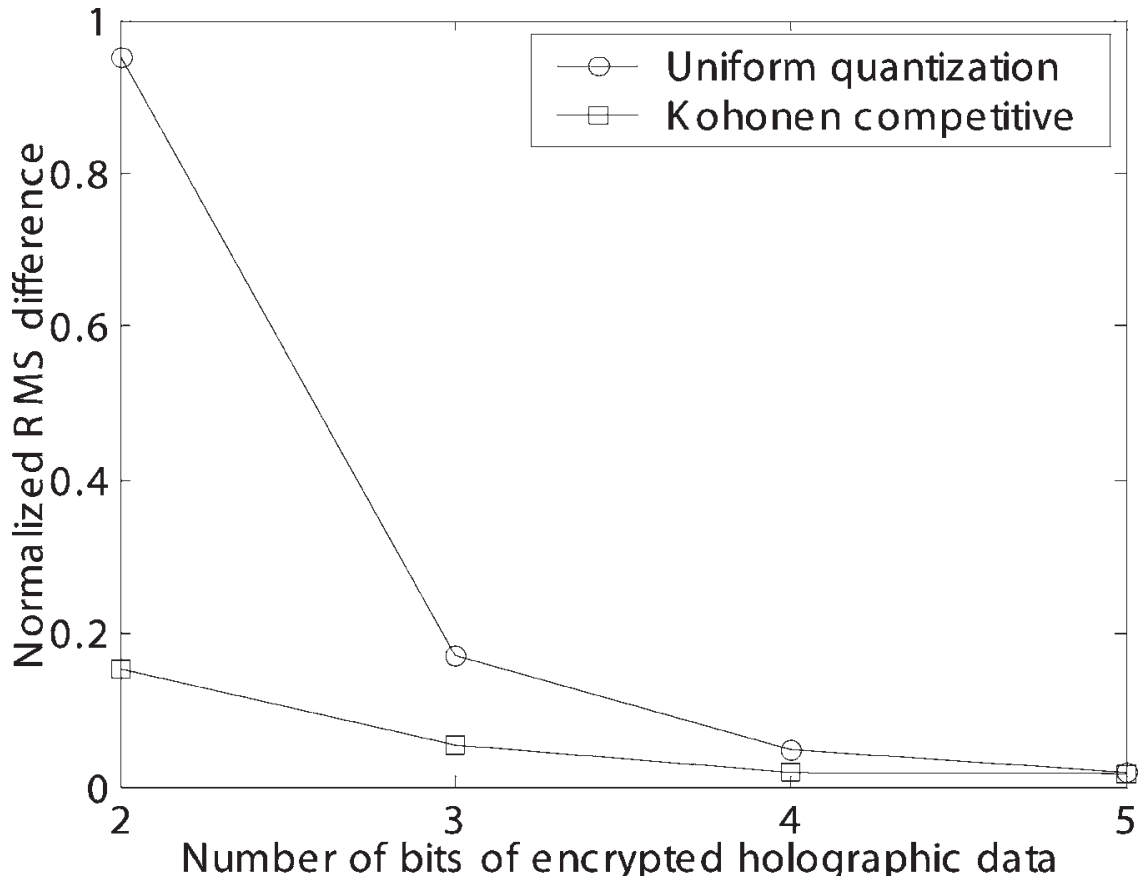

Figure 2. Intensity difference in a decrypted and reconstructed 3D object plotted against quantization level.
Figure 2 shows plots of normalized RMS difference against the number of bits of encrypted holographic data. This is data for a single hologramshowing both uniform quantization and Kohonen competitive quantization-and illustrates the consistently lower errors incurred by the latter. Nonuniform quantization not only performs significant compression itself, it also reduces the number of symbols (for Huffman) and introduces structure into the bit stream (for LZ77 and LZW) to allow algorithms to perform further compression.

The authors wish to thank Enrique Tajahuerce and Yann Frauel for use of their hologram data. The first author wishes to acknowledge support from Enterprise Ireland.

\section{difficult to remove since}

it actually carries 3D information. Its presence causes lossless data compression techniques to perform badly, so lossy compression techniques are used instead.

The encrypted complex-valued holograms can be captured using an optical setup (shown in Figure 1) based on a Mach-Zehnder interferometer architecture. ${ }^{1,3}$ We capture the complex camera-plane wavefront using phase-shift interferometry. ${ }^{6}$ These holograms are encrypted by perturbing the Fresnel diffraction of the 3D objects with the random phase mask, which contains values chosen with uniform probability from the range $[0,2 \pi)$ using a pseudorandom-number generator. The position of the phase mask is illustrated in Figure 1 and the ratio of the distances $d_{1}: d_{2}$ is 35:65. We simulated this encryption step in software, ${ }^{3}$ which gave us added flexibility and security while still allowing for future real-time optical reconstruction. Each encrypted hologram was $1024 \times 1024$ pixels.

The decryption and reconstruction of the digital hologram can be carried out optically or digitally. The hologram is propagated a distance $d_{2}$ to plane $\mathrm{P}$ and decrypted by multiplying it with the phase mask. It is reconstructed through further Fresnel propagation to focus in any chosen plane in the range $d_{1} \pm \mathrm{D}$. Like traditional holography, different angles of view of the object can be reconstructed using different windowed subsets of the hologram.

The digital holograms were first treated as binary data streams and compressed using the following lossless data compression techniques: Huffman, Lempel-Ziv (LZ77), LempelZiv-Welch (LZW), and Burrows-Wheeler (BW). An average compression ratio of 1.05 was achieved for all algorithms and for five digital holograms in our study. The poor compression ratios confirmed the lack of redundancy in the encrypted hologram data and illustrated the urgent need to explore lossy compression techniques. One such technique that we have successfully applied to 3D digital holograms is quantization. We evaluated two ANN-based non-uniform quantization techniques and found that the Kohonen competitive $\mathrm{ANN}^{5}$ performed best with our digital hologram data. In our experiments, we considered each complex value as a vector of length two and used the unsupervised ANN to locate the most suitable clusters in the encrypted digital-hologram data. The learning rates were chosen through extensive experimentation. We then quantized our encrypted holograms with the centers of these clusters. A reconstructedobject-plane RMS (root-mean-square) metric was used to quantify the quality of our decompressed and decrypted holograms.
Alison E. Shortt, Thomas J. Naughton, and Bahram Javidi*

Department of Computer Science

National University of Ireland

Maynooth, Ireland

E-mail: tom.naughton@may.ie

*Electrical and Computer Engineering

Department

University of Connecticut

CT, USA

E-mail: bahram@engr.uconn.edu

\section{References}

1. E. Tajahuerce and B. Javidi, Encrypting threedimensional information with digital holography, Appl. Opt. 39, pp. 6595-6601, 2000.

2. T. J. Naughton and B. Javidi, Compression of encrypted three dimensional objects using digital holography, Opt. Eng. 43 (10), pp. 2233-2238, 2004.

3. Y. Frauel, E. Tajahuerce, M. -A. Castro, and B. Javidi, Distortion-tolerant three-dimensional object recognition with digital holography, Appl. Opt. 40 (23), pp. 3887-3893, 2001.

4. J. W. Goodman and R. W. Lawrence, Digital image formation from electronically detected holograms, Appl. Phys. Lett. 11, pp. 77-79, 1967.

5. T. Kohonen, Self-Organizing Maps, SpringerVerlag, Berlin, 1994

6. I. Yamaguchi and T. Zhang, Phase-shifting digital holography, Opt. Lett. 22, pp. 1268-1270, 1997. 